\title{
Prepaid-Stromzähler: Erfahrungen aus der NutzerInnen-Perspektive von Haushalten in Deutschland
}

\author{
Oliver Wagner* und Julia Wiegand ${ }^{* *}$
}

\section{Zusammenfassung}

Energiearmut ist ein Phänomen, welches in Deutschland, wie auch in anderen Ländern des Globalen Nordens, in den letzten Jahren immer häufiger beobachtet werden kann. Zunehmend werden Prepaidzähler (engl. Prepayment Meter) von Energieunternehmen als Instrument eingesetzt, um KundInnen mit Zahlungsrückständen bzw. häufig auftretenden Zahlungsschwierigkeiten zu managen. Das Phänomen der Energiearmut in Deutschland ist relativ jung und damit die Anzahl der Prepaidzähler noch niedrig. Somit sind Erfahrungen in diesem Zusammenhang in Deutschland rar. Nachfolgend werden die Ergebnisse der ersten wissenschaftlichen Befragung von Haushalten mit Prepaidzählern in Deutschland (im Bundesland Nordrhein-Westfalen) analysiert. Dabei zeigt sich, dass Prepaid-Systeme für Strom für die betroffenen Haushalte mit erheblichen Alltagsveränderungen verbunden sind. Vorteilhaft ist, dass die Haushalte trotz bestehender Strom-Schulden weiterhin mit Energie versorgt werden können, dass sie über eine bessere Kostenkontrolle verfügen und dass sie Stromsparpotenziale erschließen können. Zu den wesentlichen Nachteilen zählen die hohen Kosten, der Aufwand für das Aufladen des Guthabens und dass Versorgungsunterbrechungen dennoch stattfinden, jedoch nicht erfasst werden. Insgesamt ergab die Studie eine hohe Zufriedenheit der Haushalte mit Prepaidzähler, es besteht jedoch Regulierungsbedarf seitens des Gesetzgebers in Deutschland.

Schlagwörter: Prepaidzähler, Energiearmut, Armut, Stromschulden, Stromsperre, Smart Meter, Ordnungsrahmen

\section{Prepaid meter for electricity: Experiences from the user perspective of households in} Germany

\section{Abstract}

Energy poverty is a phenomenon that can be observed more frequently in Germany in recent years, as in other countries of the global north. In this context, prepayment meters are increasingly used by energy companies as a tool to manage customers with arrears or frequent payment difficulties. The phenomenon of energy poverty in Germany is relatively young and thus the number of prepayment meters is still low. Subsequently the results of the first scientific survey of households with prepayment meters in Germany (in the federal state of North Rhine-Westphalia) are presented and analyzed. It shows that prepayment systems for electricity are associated with significant everyday changes for the affected households. Advantages are the continuation of energy supply despite existing electricity debts, a better cost control, and tapping energy-saving potential. Significant drawbacks include higher costs, the burden households have to recharge the meter, and supply disruptions take place on a larger scale but are not recorded. Despite that our survey shows a high level of satisfaction among the users. Nonetheless there is considerable need for regulation by the German legislator.

Keywords: Prepayment meter, energy poverty, poverty, electricity debt, electricity cut off, smart meters, regulatory framework

\footnotetext{
* Oliver Wagner, Wuppertal Institut für Klima, Umwelt, Energie. E-Mail: oliver.wagner@wupperinst.org.

** Julia Wiegand, Wuppertal Institut für Klima, Umwelt, Energie. E-Mail: julia.wiegand@wupperinst.org.

Wir danken den Beratungsstellen und Energieversorgungsunternehmen, die uns bei unseren Interviews unterstützt haben, und natürlich danken wir auch unseren InterviewpartnerInnen, die uns teilweise über eine Stunde ihrer Zeit geschenkt und tiefe Einblicke in ihr Privatleben gewährt haben. Zudem danken wir den anonymen Gutachtern, die mit ihren Anregungen und Hinweisen wesentlich zu einer Verbesserung dieses Artikels beigetragen haben.
} 


\section{Hintergrund}

Laut dem jüngsten Armutsbericht des Paritätischen Wohlfahrtsverbands erreicht die Armutsquote im Jahr 2015 die Rekordhöhe von 15,7 Prozent, was bedeutet, dass aktuell rund 13 Millionen Menschen in Deutschland unter der Einkommensarmutsgrenze leben (Der Paritätische Gesamtverband 2017: 9). Zahlreiche einkommensarme Haushalte und diejenigen, die nahe der Armutsgrenze leben, leiden unter den in Deutschland in den letzten Jahren gestiegenen Stromkosten sowie einem Verlust an Realeinkommen. Insbesondere hinsichtlich der verzögerten Anpassung der Regelsätze von Haushalten, die staatliche Transferleistungen erhalten, herrscht weitgehend Konsens darüber, dass diese mit den gestiegenen Energiekosten in den letzten Jahren nicht Schritt gehalten hat (Strünck 2017: 5). Die Energiekosten für Strom und Heizung stellen für einkommensarme Haushalte einen im Verhältnis zum verfügbaren Gesamtbudget großen Anteil ihrer Fixkosten dar. Neben dem geringen Einkommen sind es auch Faktoren wie defekte bzw. veraltete Elektrogeräte, ineffiziente Heizungen oder sanierungsbedürftige Wohnungen, die hohe Energiekosten verursachen (Stückler 2015: 3). Der Begriff „Energiearmut“, welcher aus dem Englischen für „energy poverty“ übernommen wurde (Schaffrin et al. 2017: 2; Kopatz et al. 2010: 6) und für den es im deutschsprachigen Raum sowie den meisten EU-Staaten noch keine einheitliche Definition gibt (E-Control 2013: 7), hat in den letzten Jahren einen Bedeutungsgewinn als eigenes Problemfeld im Rahmen der Armuts- und Sozialforschung erfahren. Strünck kommt zu dem Ergebnis, dass rund 21,5 Prozent (8,5 Millionen) aller Haushalte in Deutschland (39,4 Millionen) energiearm sind und liegt mit seinen Ergebnissen leicht über den Berechnungen der Verbraucherzentrale NRW aus dem Jahr 2008, wonach ca. 20 Prozent als energiearm bezeichnet werden können, weil sie mehr als 13 Prozent ihres verfügbaren Einkommens für Energiekosten ausgeben müssen (Verbraucherzentrale NRW 2008). Dabei sind nicht nur Haushalte betroffen, die Transferleistungen beziehen. Die Gruppe der GeringverdienerInnen leidet ebenso unter Energiearmut. Selbst in der Gruppe der Haushalte mit Erwerbseinkommen liegt die Quote energiearmer Haushalte bei 14,7 Prozent (Strünck 2017: 11). Unabhängig davon, wie groß der Anteil von Energiearmut betroffener Haushalte ist, muss auch in Zukunft damit gerechnet werden, dass Energiekostenbelastungen und deren ungleiche Verteilung in der
Gesellschaft weiter zunehmen werden, wodurch der Druck auf Entscheidungsträger wieder steigen wird, sich mit den sozialen Konsequenzen der Energiewende intensiver zu beschäftigen (Schaffrin et al. 2017: 2).

In der Folge steigender Energiekosten und zunehmender Einkommensarmut hat die Anzahl von Stromsperren in Deutschland deutlich zugenommen. Nach Angaben der Bundesnetzagentur wurden im Jahr 2015 aufgrund von Zahlungsrückständen insgesamt knapp 359.000 Unterbrechungen der Stromversorgung durch die Energieunternehmen veranlasst. Um solche Stromsperren zu vermeiden bzw. eine Wiederaufnahme der Stromversorgung zu ermöglichen, bieten immer mehr Versorgungsunternehmen ihren KundInnen einen Prepaidzähler an. Gemäß dem Prinzip des PrepaidHandys wird Guthaben aufgeladen, welches für den Stromverbrauch im Haushalt zur Verfügung steht.

Anbieter von solchen Prepaid-Systemen ist in der Regel der jeweilige Grundversorger, also jenes Energieversorgungsunternehmen, welches die meisten HaushaltskundInnen im jeweiligen Ortsverteilnetz mit Strom bzw. Gas beliefert. Die Rechte und Pflichten, die sich aus dem Status des Grundversorgers für Strom ergeben, regelt in Deutschland die Stromgrundversorgungsverordnung (StromGVV). Darin werden die Bedingungen geregelt, zu denen HaushaltskundInnen mit Elektrizität versorgt werden müssen. Die zu erfüllenden Voraussetzungen, um dem Grundversorger eine Zählersperre zu erlauben, sind in $\$ 19$ (Unterbrechung der Versorgung) geregelt. Demnach ist bereits ein geringfügiger Zahlungsverzug von 100 Euro ausreichender Grund, um eine Zählersperre zu veranlassen und damit die Versorgung zu unterbrechen.

\subsection{Grundbedürfnis Strom}

Die häusliche Nutzung von Elektrizität ist ein zentraler Bestandteil sozialer Teilhabe und notwendige Bedingung, um grundlegenden Bedürfnissen nachgehen zu können. Erschwingliche, zuverlässige Versorgungsleistungen zählen zu den Grundbedürfnissen eines würdevollen Lebens in modernen Gesellschaften (Brunner et al. 2012: 57). Doch die tägliche Dusche, die Zubereitung warmer Mahlzeiten sowie die Nutzung einer Waschmaschine kann für einkommensarme Haushalte zum Luxus werden.

In ihrem jährlichen Bericht über die Entwicklung der Elektrizitäts- und Gasmärkte in Deutschland erfassen Bundeskartellamt und Bundesnetzagentur Stromsperren von HaushaltskundInnen. Aufgrund von 
Stromschulden werden demnach jährlich über 300.000 Haushalte in Deutschland von der Energieversorgung getrennt, mit steigender Tendenz. 2011 zählten noch etwa $312.000 \mathrm{zu}$ den betroffenen Haushalten (Bundeskartellamt/Bundesnetzagentur 2012: 125) und 2015 waren es bereits rund 359.000 (Bundeskartellamt/Bundesnetzagentur 2016: 25). Als Ursachen gelten steigende Energiepreise, stagnierende oder sinkende Einkommen, Arbeitslosigkeit sowie die lediglich schleppende Umsetzung von Energieeffizienzmaßnahmen in Wohngebäuden (Brunner et al. 2012: 53). Durch lange Abrechnungszeiträume und den Mangel an zwischenzeitlichen Rückmeldungen bezüglich des „Stromkontostands“ entstehen oft hohe Nachzahlungen, die vor allem bei einkommensschwachen Haushalten Schulden verursachen, die sie nicht oder nur schwer begleichen können (Brunner et al. 2012: 56). In vielen Haushalten sorgen multiple finanzielle Belastungen für deren benachteiligte Lage und der Kreis der von Energiearmut gefährdeten Menschen erweitert sich stetig (Großmann 2017: 75). Wie eingangs dargestellt, zählen längst auch Berufstätige dazu, deren Arbeitseinkommen nur knapp über der Bemessungsgrenze für Wohngeld und Kinderzuschlag liegt (Kopatz 2013: 41). Diese Haushalte sind besonders stark von der Zunahme der Ausgabenanteile für Strom am verfügbaren Einkommen betroffen, welche seit 2003 feststellbar ist. Vor allem in den untersten Einkommensgruppen ist ein starker Anstieg der Ausgabenanteile zu beobachten, während für obere Einkommensgruppen die Preissteigerungen kaum ins Gewicht fallen (Heindl \& Löschel 2016: 10).

\subsection{Das Prepaid-Prinzip}

Stromzähler mit einer Prepaid-Funktion werden von einigen Sozialverbänden, Energieversorgungsunternehmen und WissenschaftlerInnen als Lösung angesehen, um StromkundInnen trotz finanzieller Schwierigkeiten weiterhin mit Elektrizität zu versorgen, eine Stromsperre zu vermeiden und die angehäuften Stromschulden in den Griff zu bekommen (Kopatz 2013: 196). Das Prinzip ist hierbei ähnlich wie bei Prepaid-Mobiltelefonen: KundInnen laden ihren Stromzähler über einen Chip mit einem Geldbetrag auf, den sie für ihren Stromverbrauch zur Verfügung haben. Der aufgeladene Betrag verringert sich in dem Maß, wie Strom für elektrische Anwendungen verbraucht wird. Ist das Guthaben aufgebraucht, kann in der Regel auch kein weiterer Strom genutzt werden. Somit wird die Verantwortung (Berger 2017: 403), aber auch die Möglichkeit einer regelmäßigen Kontrolle des Energiekonsums auf die VerbraucherInnen übertragen.

Oft ist die Nutzung eines Prepaidzählers mit zusätzlichen Kosten für die StromkundInnen verbunden und stellt nicht automatisch eine finanzielle Entlastung dar. Denn die Mehrkosten für die Zähler sowie die aufgelaufenen Altschulden müssen über den aktuellen Strombezug der KundInnen beglichen werden. Somit schlägt der Sachverständigenrat für Umweltfragen (SRU) vor, dass die Kosten zur Installation eines Prepaid-Zählers bei finanzschwachen Haushalten auf Antrag durch die öffentliche Hand bzw. die Leistungsträger übernommen werden sollten. Außerdem sollte insbesondere bei Wohnungen einkommensschwacher Haushalte darauf geachtet werden, im Zuge des bevorstehenden Rollouts intelligenter Stromzähler (Smart Metering) gesetzgeberisch festzuschreiben, dass die neuen Zähler eine Prepaid-Funktion ermöglichen (Sachverständigenrat für Umweltfragen 2016: 207).

\subsection{Erfahrungen mit Prepaidzählern in Großbri-} tannien

In Großbritannien verbreitet sich die Nutzung von Prepaidzählern bereits seit über 20 Jahren. Die Anzahl der Prepaid-NutzerInnen hat sich dort von 7 Prozent im Jahr 1996 auf 16 Prozent im Jahr 2015 mehr als verdoppelt (Hodges et al. 2016: 2). Bis heute nutzen mehr als vier Millionen Haushalte in Großbritannien Prepaidzähler für Strom (European Commission 2016: 355). Im Vergleich dazu ist die in Deutschland installierte Anzahl an Prepaidzählern mit etwa 19.400 eher gering und liegt damit bei unter 0,05 Prozent (Bundeskartellamt/Bundesnetzagentur 2016: 26). Grund für den Anstieg in Großbritannien ist die stark gestiegene Armut. Die Autoren einer Studie der London School of Economics sortieren nahezu jeden zweiten britischen Haushalt in eine der drei untersten von sieben Klassen ein. 15 Prozent zählen demnach zum Prekariat mit einem durchschnittlichen Jahreseinkommen von weniger als 8000 Pfund (das entspricht weniger als 10.000 Euro), ohne Ersparnisse oder Grundbesitz, darüber hinaus seien sie sozial und kulturell abgehängt (vgl. Savage et al. 2014).

Britische Studien zeigen, dass Prepaid-KundInnen verstärkt Haushalte sind, die gegenüber Energiearmut besonders vulnerabel sind. Dennoch müssen gerade diese Haushalte durch den Prepaidzähler oft höhere Kosten tragen und haben weniger Auswahl bei Stromtarifen. Aus diesem Grund hat die britische Behörde 
für Gas- und Elektrizitätsmärkte „ofgem“ (Office of Gas and Electricity Markets) kürzlich Pläne angekündigt, gemeinsam mit der Regierung einen Gesetzesentwurf zur Einführung einer Preisobergrenze für Standardtarife einzubringen. Eine Preisobergrenze für Stromtarife bei Vorkassezählern (der sogenannte „safeguard tariff“) wurde in Großbritannien bereits erfolgreich eingeführt, obwohl dies ein erheblicher Eingriff in den Markt ist. Doch laut ofgem funktioniert der Markt gerade für vulnerable Haushalte nicht, was staatliche Eingriffe erforderlich macht (ofgem 2017).

\subsection{Stromsperren in Deutschland}

Es liegt in der technischen Eigenart der Prepaidzähler, dass KundInnen mit einem solchen Zähler exponiert gegenüber dem hauptsächlichen Nachteil solcher Systeme sind: dem Risiko der Selbstsperre. Wenn das Guthaben aufgebraucht ist, endet für gewöhnlich die Stromversorgung, was de facto einer Selbstsperre entspricht. Stromsperren werden in Deutschland jedoch nur von Nicht-Prepaid-KundInnen statistisch erfasst, indem die Daten der durch den Grundversorger durchgeführten Zählersperren erhoben werden. Gemäß Artikel 3 (7) und (8) der Elektrizitätsmarkt Direktive (2009/72/EC) müssen angemessene Maßnahmen gegen Energiearmut von vulnerablen VerbraucherInnen ergriffen werden. Aus diesem Grund erheben die Mitgliedsstaaten der Europäischen Union Stromsperren von Nicht-Prepaid-KundInnen und fassen diese regelmäßig in Monitoring-Berichten nach einheitlich definierten Kriterien zusammen. In Deutschland ist damit die Bundesnetzagentur betraut, die jährlich gemäß \$63 Energiewirtschaftsgesetz einen Bericht der Europäischen Kommission und der Europäischen Agentur für die Zusammenarbeit der Energieregulierungsbehörden vorlegt. In diesem Bericht wird auch die Zahl der Sperrungen von HaushaltskundInnen in der Grundversorgung erfasst. Laut Monitoringbericht 2016 wurden rund 6,3 Mio. Sperrandrohungen von den Lieferanten gegenüber zumeist grundversorgten HaushaltskundInnen ausgesprochen, von denen 1,6 Mio. in eine Unterbrechungsbeauftragung beim zuständigen Netzbetreiber mündeten, wovon letztendlich rund 359.000 Sperrungen vollzogen wurden. Davon waren 331.273 HaushaltskundInnen in der Grundversorgung, die restlichen außerhalb (Bundeskartellamt/ Bundesnetzagentur 2016: 25). Über diese Erhebungen hinaus werden jedoch - anders als beispielsweise in Großbritannien - keine vergleichbaren Bemühungen unternommen, das Ausmaß und die Treiber der Selbstabschaltung bei Prepaid-KundInnen zu erfassen.

Durch einen breiteren Einsatz von Prepaidzählern könnte die Zahl der durch Energieversorgungsunternehmen veranlassten Stromsperren reduziert und weniger Menschen vom Grundbedürfnis der Stromversorgung ausgeschlossen werden. Dennoch soll damit keinesfalls eine Verschleierung des Problems der Energiearmut betrieben oder lediglich ein scheinbarer Erfolg im Kampf gegen Energiearmut verzeichnet werden. Somit ist es von großer Bedeutung, empirisch gesicherte Kenntnisse über das Ausmaß und die Konsequenzen der Nutzung von Prepaidzählern und insbesondere der Selbstabschaltung zu generieren.

Über den Umgang mit Prepaidzählern aus der NutzerInnen-Perspektive ist in Deutschland bislang nur wenig bekannt. Der vorliegende Beitrag beinhaltet Ergebnisse einer empirischen Studie in mehreren Städten in Nordrhein-Westfalen und soll einen Beitrag dazu leisten, die bestehende Wissens- und Forschungslücke zu schließen.

\subsection{Stand der Forschung}

Einige Studien aus Großbritannien betrachten das Ausmaß und die Treiber der Selbstabschaltung und werden von Brutscher zusammengefasst (Brutscher 2012: 2). Demnach variieren die Ergebnisse der Studien bedeutend. Es ist unklar, ob die Selbstabschaltung ein weit verbreitetes Phänomen ist und wie hoch die Bedeutung finanzieller Zwänge im Zusammenhang mit Selbstsperren ist (ebd.). Ein Vorteil von Prepaidzählern besteht in der zeitnahen Abrechnung, womit eine stärkere Kostenkontrolle einhergeht und weshalb Prepaidzähler von der Mehrheit der VerbraucherInnen als positiv angesehen werden (European Commission 2016: 355).

Inzwischen steigt EU-weit die Anzahl an Prepaidzählern. Einige der oben genannten Probleme, wie höhere effektive Kosten für die Nutzung von Prepaidzählern, wurden ebenfalls in den Niederlanden identifiziert (European Commission 2016: 355f). Nach Angaben eines Verbraucherverbandes werden einkommensarme Stromkunden für gewöhnlich in teurere Tarife eingestuft. Entweder, weil eine Kaution für den Prepaidzähler erforderlich war, oder schlicht deswegen, weil für den Gebrauch des Zählers eine höhere Grundgebühr verlangt wurde.

Dies ist auch in Neuseeland der Fall. O'Sullivan et al. (2013) stellten fest, dass Prepaidzähler mit höheren 
Kosten für die Haushalte verbunden sind als andere Bezahlformen. Dennoch konnte wie in Großbritannien eine große Zufriedenheit mit der Nutzung des Zählers festgestellt werden, was vorwiegend auf die Kostenkontrolle beim Stromverbrauch zurückzuführen ist. Nach Faruqui et al. (2010) können darüber hinaus durch Prepaidzähler in Kombination mit in der Wohnung installierten Displays Einsparungen des Stromverbrauchs von bis $\mathrm{zu} 14$ Prozent erreicht werden. Entsprechende Informationen der NutzerInnen hinsichtlich Einsparpotenzialen, Zufriedenheit und Zusatzkosten durch Prepaidsysteme in Deutschland lagen bislang nicht vor.

Eine österreichische Studie (Berger 2011) kommt auf Basis von ExpertInneninterviews zu dem Ergebnis, dass Prepaidzähler als praktische Lösung empfunden werden, um die Spirale aus Mahnungen und potenzieller Abschaltung zu durchbrechen. Der Prepaidzähler dient demnach auch zur Disziplinierung „problematischer KundInnen“. Als Defizit wurde hier erkannt, dass die mit einem solchen Zähler verbundenen Modalitäten vielen KundInnen nicht bekannt sind. Demnach wissen die Haushalte oft nicht, wie hoch der Betrag ist, den sie für die Rückzahlung von Altschulden aufbringen, und welcher Betrag zum Stromverbrauch genutzt werden kann. In der vor einer gesetzlichen Neuregelung durchgeführten Studie konnte seinerzeit festgestellt werden, dass die Ratenanteile zur Schuldentilgung im Monat oft sehr hoch angesetzt sind und dass das Aufladen der Chipkarte für den Zähler vor allem in ländlichen Räumen mit einem besonders hohen Mobilitätsaufwand verbunden ist. Zudem wurde festgestellt, dass bei manchen Energieunternehmen hohe Gebühren für Prepaidzähler genommen wurden und mit einer stigmatisierenden, beschämenden oder psychisch belastenden Wirkung durch Prepaidzähler zu rechnen sei (Berger 2011: 44).

Für Deutschland stellt Heindl (2014) fest, dass steigende Energiepreise eine Bedrohung für Haushalte mit geringerem Einkommen darstellen und Preiserhöhungen, die beispielsweise durch eine Erhöhung der realen Preise für fossile Brennstoffe sowie durch gestiegene Umlagen entstehen, diese Gefahr erhöhen. Haushalte benötigten Zeit, um sich an Preisänderungen durch eine Erhöhung der Energieeffizienz anzupassen, wovon jene mit niedrigeren Einkommen besonders betroffen sind. Aufgrund von Budgetbeschränkungen haben sie weniger Möglichkeiten, elektrische Geräte schnell zu ersetzen oder gar in energieeffizientere Wohnungen umzuziehen (ebd.).
Brischke \& Thomas (2014) haben im Rahmen ihrer Studie drei prinzipielle Ansatzpunkte identifiziert, die zu einer Energiesuffizienz führen: Reduktion, Substitution und Anpassung.

Reduktion meint die rein quantitative Verringerung des Technikgebrauchs, ohne den Nutzen qualitativ zu verringern. Substitution zielt auf den Ersatz von Gewohnheiten oder Gerätschaften durch weniger energieintensive, wie das Wäschetrocknen auf der Wäscheleine statt im Wäschetrockner. Anpassung erfordert eine technische Ausstattung mit Geräteart, Größe und Funktionen, welche dem tatsächlichen Bedarf des Haushalts entspricht (ebd.). Ob und in welcher Art Prepaidzähler hier Veränderungen anregen, war bislang nicht bekannt.

Der verstärkte Einsatz von Prepaidzählern muss auch vor dem Hintergrund der sozio-technischen Transformation des Energiesystems verstanden werden (Berger 2017: 404).

Berger sieht das Verhältnis von StromkundInnen zu Energieversorgungsunternehmen durch Prepaidzähler „neu konfiguriert“. Der Prepaidzähler fungiere als Vermittler zwischen den beiden Akteursgruppen und spiegele deren Machtverhältnis wider. Prepaidzähler müssen darüber hinaus im Zusammenhang mit neoliberaler Wirtschaftspolitik und der Liberalisierung der Energiewirtschaft in Europa betrachtet werden. Der Prepaidzähler sichert das marktbasierte Austauschverhältnis für die Dienstleistung „Energie“, nachdem das Vertrauen in die Zahlungsfähigkeit der einkommensschwachen KundInnen nicht mehr vorhanden ist (ebd.).

Einen Überblick der politischen Ansätze verschiedener EU-Mitgliedsstaaten zur Bekämpfung von Energiearmut gibt eine aktuelle Studie von Strünck (2017), die im Auftrag der Friedrich-Ebert-Stiftung erstellt wurde. Strünck kommt zu dem Ergebnis, dass die meisten Maßnahmen in Europa darauf abzielen, energiearme Haushalte finanziell zu unterstützen. Besonders häufig sind demnach klassische Instrumente der sozialen Sicherung in Form von Zuschüssen oder Grundsicherungsleistungen anzutreffen. Aber auch sogenannte Sozialtarife für verschiedene Energieträger sind ein in der EU weit verbreitetes Mittel (Strünck 2017). Auch in Deutschland wurde in der Vergangenheit bereits die Diskussion um die Einführung eines solchen Tarifs geführt. Da allerdings keiner der im Raum stehenden Vorschläge einer solchen Tarifgestaltung die gewünschte zielgruppenspezifische Entlastungswirkung herbeiführen kann, ohne gleichzeitig 
erheblich unerwünschte Nebeneffekte aufzuweisen (Wagner et al. 2008: 38), wurden erste Überlegungen schnell wieder fallen gelassen. Heute zeigt sich, dass auch in Ländern, die einen solchen Tarif eingeführt haben, wie beispielsweise Frankreich, Sozialtarife die Zielgruppe der einkommensschwachen Haushalte weitgehend verfehlen (Strünck 2008: 24).

\section{Methodik}

Ziel unserer Studie war es, das NutzerInnenverhalten von Haushalten mit Strom-Prepaidzählern in Deutschland zu untersuchen. Dabei stand im Vordergrund, wie VerbraucherInnen mit dieser Art des Stromerwerbs umgehen und welche Effekte der Gebrauch eines Prepaidzählers auf den Stromverbrauch hat. Im Rahmen dieser Studie, welche im Auftrag des Ministeriums für Umwelt, Landwirtschaft, Natur- und Verbraucherschutz des Landes Nordrhein-Westfalen (NRW) durchgeführt wurde, wurden im Zeitraum von Herbst bis Winter 2016/17 semi-strukturierte Interviews mit derzeitigen NutzerInnen von Prepaidzählern in mehreren Städten Nordrhein-Westfalens, schwerpunktmäßig im Ruhrgebiet, durchgeführt (Kopatz et al. 2017).

Diese Methode wurde gewählt, da Leitfadeninterviews vor allem ein geeignetes Instrument sind, um explorativ eingesetzt zu werden und Hypothesen in einem weithin unerforschten Gebiet zu entwickeln (Schnell et al. 1989: 295; Stier 1999: 189), da der Forschungsgegenstand (hier die betroffenen Haushalte mit einem Prepaidzähler) noch nicht in allen Dimensionen klar umrissen ist. Durch das Fehlen von Vorarbeiten und die geringe Fallzahl kamen standardisierte Forschungsmethoden allein nicht in Frage. Der Leitfaden, der auch semi-standardisierte Elemente mit konkreten Antwortvorgaben beinhaltete, diente vor allem als Gerüst, um eine vergleichende Datenerhebung sowie eine Ergebnisdarstellung der geführten Interviews zu ermöglichen (Bortz \& Döring 1995: 289).

Die semi-strukturierten Interviews bilden die Datenbasis für die Erfahrungen von 40 Haushalten. In Bezug auf die hier behandelte Fragestellung ist Nordrhein-Westfalen als das einwohnerstärkste Bundesland Deutschlands besonders interessant. Der Paritätische Wohlfahrtsverband hat in seinem sogenannten Armutsbericht festgestellt, dass NRW und insbesondere das Ruhrgebiet eine „armutspolitische Problemregion“ ist (Der Paritätische Gesamtverband 2017: 17). NRW belegt im Vergleich den letzten Platz unter den westdeutschen Flächenländern. Auffällig ist zudem, dass im Zehnjahresvergleich die Armutsquote von 14,4 Prozent auf 17,5 Prozent deutlich anstieg und damit die bundesweit höchste Zunahme verzeichnet wurde (Der Paritätische Gesamtverband 2017: 12). Gleichzeitig gibt es in NRW im Rahmen eines Landesmodellprojekts auch besondere Bemühungen sowie politische und unternehmerische Initiativen, etwas gegen Energiearmut zu unternehmen (Schöllgen \& Kosbab 2017: 475 ff.).

Die Interviews enthielten sowohl quantitative Anteile über Mengen und Kosten des Stromverbrauchs als auch qualitative Fragestellungen zur Perspektive der VerbraucherInnen, zu den Problemen im Zusammenhang mit Prepaidzählern sowie Verbesserungsvorschläge. Der Interviewleitfaden wurde in Anlehnung an Studien aus anderen Ländern verfasst, um trotz der geringen Fallzahl eine Vergleichsgröße zu haben.

Der Kontakt zu den InterviewpartnerInnen war trotz einer in Aussicht gestellten Aufwandsentschädigung in Höhe von 20 Euro pro Befragung schwierig herzustellen, konnte jedoch mit Unterstützung von Energieversorgungsbetrieben, welche die Nutzung von Prepaidzählern anbieten, sowie Sozialorganisationen und Schuldnerberatungen hergestellt werden. Das Problem des Zugangs zu den betroffenen Haushalten stellt sich auch in anderen Ländern gleichermaßen dar. So wurden beispielsweise in Neuseeland, Großbritannien und der Steiermark auf Basis semi-strukturierter Interviews qualitative Erhebungen mit NutzerInnen von Prepaid-Stromzählern durchgeführt, wobei in Neuseeland zwölf (O'Sullivan et al. 2015), in Großbritannien 30 (Simpson et al. 2016) und in der Steiermark zehn (Berger 2011) Interviews geführt wurden. Aufgrund der geringen Fallzahl erwies sich in unserer, wie auch in den genannten anderen Studien, eine tiefgehende statistische Analyse als nicht sinnvoll durchführbar. Die vorliegende Analyse beschränkt sich daher vor allem auf eine Darstellung der Häufigkeitsverteilungen. Die Ergebnisse sind aufgrund der geringen Fallzahl nicht repräsentativ, zeigen aber deutliche Tendenzen auf. Die Interviews wurden persönlich mit den PrepaidNutzerInnen in den Kundencentern der Energieversorger sowie im Rahmen von Hausbesuchen geführt. Die semi-strukturierten Interviews ermöglichten es, wertvolle Zusatzinformationen $\mathrm{zu}$ erhalten, die in einem starren Korsett eines voll strukturierten Fragebogens nicht hätten aufgenommen werden können. So konnten teilweise detaillierte Einblicke in den Alltag von Prepaid-nutzenden Haushalten gewonnen und Probleme erkannt werden, die wir bei der Entwicklung des 
Abbildung 1: Häufigkeiten von Angaben zu Reaktionen auf den Prepaidzähler; Zufriedenheit mit Prepaidzähler

Reaktionen aus dem Umfeld

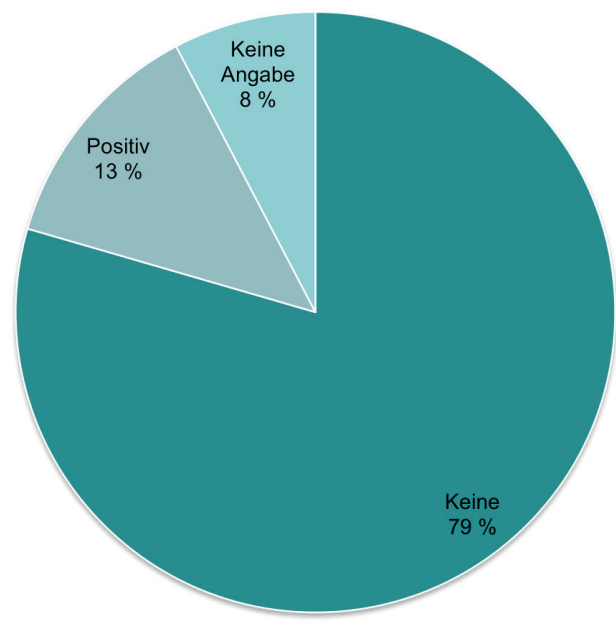

Quelle: Kopatz et al. 2017.

Interviewleitfadens nicht bedacht hatten. Ein Beispiel hierfür ist der Umstand, dass Energieversorgungsunternehmen durch einen Prepaidzähler im Falle eines Verbraucherinsolvenzverfahrens (in Österreich: Schuldenregulierungsverfahren) der KundInnen gegenüber anderen Gläubigern besser gestellt sind, da sie Altschulden über den Prepaidzähler eintreiben können. Dies ist anderen Gläubigern nicht möglich, zudem läuft dies dem Zweck der Insolvenz, nämlich dem Ablegen der Schulden bei Sicherung eines Existenzminimums, zuwider.

\section{Ergebnisse der Interviews}

Aus den NutzerInnenerfahrungen von Haushalten mit Prepaidzählern konnten Erkenntnisse über den Status quo der verschiedenen in Nordrhein-Westfalen genutzten Prepaid-Systeme sowie für deren Weiterentwicklung und Verbesserung gewonnen werden.

Zufriedenheit mit Prepaidzählern aus der NutzerInnen-Perspektive: Ein gängiges Argument gegen den Einsatz von Prepaidzählern basiert auf der Annahme, dass Prepaidzähler in Mietshäusern eine soziale Stigmatisierung für die Betroffenen bedeuten würden, was solche Zähler unattraktiv mache (Heindl \& Löschel 2016). Dies konnte jedoch mit dieser Studie widerlegt werden. Die befragten NutzerInnen sind eher mit positiven Reaktionen (13 Prozent) konfrontiert, falls es überhaupt zu Reaktionen kommt, denn 79 Prozent gaben an, keine Reaktionen aus dem Umfeld bemerkt
Zufriedenheit mit dem Prepaid-Zähler

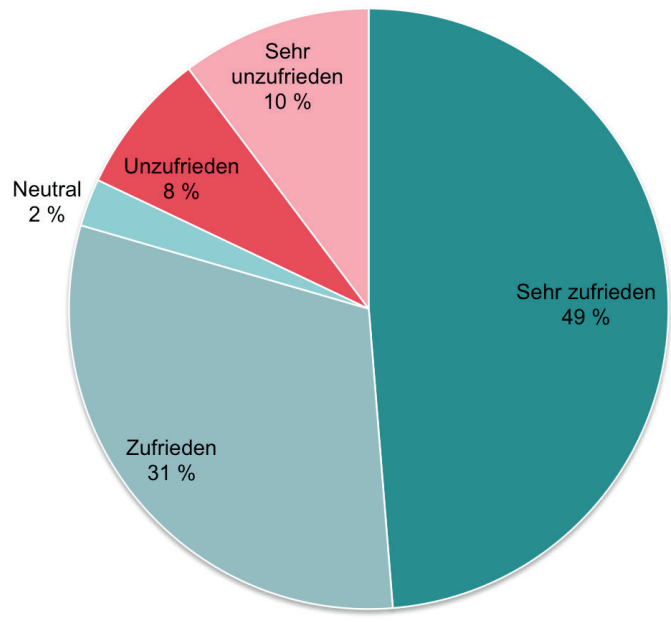

zu haben und weitere 8 Prozent konnten uns die Frage nicht beantworten (Abb. 1, links).

Aus der Befragung nach der allgemeinen Zufriedenheit mit dem Prepaidzähler (Abb. 1, rechts) geht hervor, dass eine deutliche Mehrheit von 8o Prozent der befragten NutzerInnen zufrieden oder sogar sehr zufrieden sind. 10 Prozent gaben an, sie seien sehr unzufrieden mit dem Zähler und 8 Prozent sind unzufrieden damit. Ferner ergab unsere Befragung, dass sich 69 Prozent der Befragten erneut für einen Prepaidzähler entscheiden würden. Weiterempfehlen würden einige der Befragten einen Prepaidzähler nur, wenn sich dadurch bei bestehenden Energieschulden eine Stromsperre vermeiden lässt. Somit zeigt sich zwar eine Tendenz zur NutzerInnen-Zufriedenheit, jedoch entstanden aus den Kritiken der Befragten zahlreiche Anknüpfungspunkte für Verbesserungen. Insbesondere technisch-organisatorische Aspekte wurden häufig kritisiert.

Der Umgang mit Altschulden wurde in drei Fällen (8 Prozent) als verbesserungswürdig eingestuft. Dies verdeutlicht, dass trotz der Ursache für den Einbau des Prepaidzählers, nämlich nicht beglichener Verbindlichkeiten, seitens der Haushalte eine hohe Bereitschaft besteht, auch den Zahlungsverpflichtungen aus vorhandenen Altschulden nachkommen zu wollen. Ansonsten wäre dieser Aspekt häufiger beklagt worden. Von Seiten der NutzerInnen wird die Vorgehensweise der Energieversorger kaum als problematisch betrachtet, da die Erleichterung über die 
Abbildung 2: Häufigkeiten der Selbstsperren von PrepaidnutzerInnen

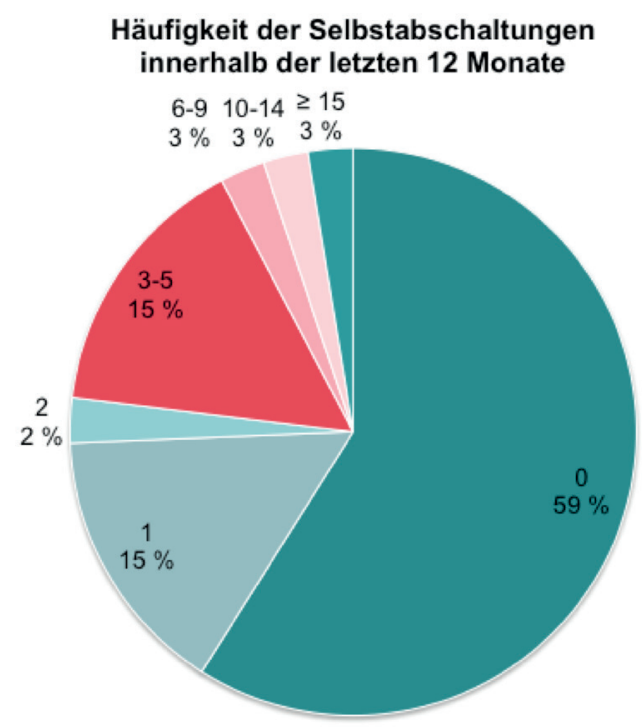

Quelle: Kopatz et al. 2017.

umgangene Stromsperre und die neugewonnene subjektive Kostentransparenz überwiegen. Hinzu kommt, dass Prepaid-KundInnen in den meisten Fällen nicht den Stromtarif oder gar den Energieversorger wechseln können. Sie sind gezwungen, bei ihrem Grundversorger zu bleiben (Kopatz et al. 2017), weil alternative Anbieter in der Regel vor Vertragsabschluss Auskünfte über die Kreditwürdigkeit bzw. Zahlungsfähigkeit neuer KundInnen einholen.

\subsection{Aufladen des Prepaidzählers und Selbstsper-} ren

Die Aufladung der Prepaidzähler erfolgt durch die NutzerInnen in den Kundencentern der Energieversorger. Meist fungiert eine Chipkarte oder ein digitaler Schlüssel als Transfermedium des Geldbetrags zum Prepaidzähler zu Hause. Die Studie ergab, dass die Befragten als Nachteil des Prepaidzählers am häufigsten den hohen Aufwand für das Aufladen nannten ( 38 Prozent). Der Aufladevorgang ist an die Öffnungszeiten der Kundencenter gebunden, was einen weiteren häufig genannten Kritikpunkt der NutzerInnen darstellt (23 Prozent). An den Befragungsorten bestand während des Befragungszeitraumes keine Möglichkeit, die Aufladung über Online-Bezahlsysteme, SMS-TANVerfahren oder beispielsweise an Kiosken mit langen Öffnungszeiten durchführen zu können. Nach Anga- ben der NutzerInnen besteht dringender Bedarf an zusätzlichen und jederzeit zugänglichen Auflademöglichkeiten, die nach eigenen Vorschlägen der Befragten im Bereich von Geldautomaten installiert werden sollten. Eine ortsunabhängige Aufladung mittels SMSTAN-Verfahren wurde kürzlich bei den Stadtwerken Duisburg eingeführt (Stadtwerke Duisburg 2017).

Als störend wurde empfunden, dass eine Selbstsperre durch aufgebrauchtes Guthaben auch außerhalb der Öffnungszeiten der Kundencenter erfolgt. Der Umgang mit Selbstsperren ist durch die jeweiligen Energieversorger individuell geregelt. Bei manchen Versorgern kann es außerhalb der Öffnungszeiten nicht zu einer Versorgungsunterbrechung kommen, bei anderen hingegen ist eine Selbstsperre jederzeit möglich. Die Befragungen haben gezeigt, dass Selbstsperren vor allem in der Anfangszeit nach Installation eines Prepaidzählers vorkommen. Im Sinne des VerbraucherInnenschutzes sollten Häufigkeiten und Umfang der Selbstsperren anonym erfasst werden. So kann die Entwicklung der Versorgungslage der Haushalte ermittelt werden und die Ergebnisse können in Monitoringberichte einfließen.

Mehr als die Hälfte der Befragten (62 Prozent) gaben an, bislang keine negativen Erfahrungen mit Selbstsperren gemacht zu haben. Dabei wurde nach der für die Befragten schlimmsten Erfahrung im Zusammenhang mit Selbstsperren gefragt, was jedoch keine besondere Auffälligkeit hervorbrachte.

Ein weiterer Kritikpunkt galt technischen Problemen abhängig vom installierten Zählertyp. Beispielsweise funktionierte oftmals die Aufladung nicht, sodass für die NutzerInnen erneut der Aufwand des Aufladeverfahrens mit dem Weg zum Kundencenter entstand. Auch stellt sich der oftmals erforderliche Mindestbetrag für die Aufladung als problematisch dar.

Nach den Vorteilen des Prepaidzählers gefragt, nannten die Befragten am häufigsten die aus ihrer Perspektive bestehende Kostentransparenz.

\subsection{Kostentransparenz}

Fast alle befragten Haushalte (97 Prozent) gaben an, die Höhe ihrer wöchentlichen oder monatlichen Stromausgaben zu kennen. Somit kennen NutzerInnen von Prepaidzählern eher ihre Stromkosten, als dies im Bundesdurchschnitt (87 Prozent) der Fall ist (PWC 2015). Dies könnte auf die jeweilige Beschäftigung mit dem Stromverbrauch zurückzuführen sein. 79 Prozent der Befragten gaben an, sich seit dem Einbau des 
Abbildung 3: Arbeitspreise für Strom mit Prepaidzähler; Beurteilung des Stromverbrauchs einzelner Geräte

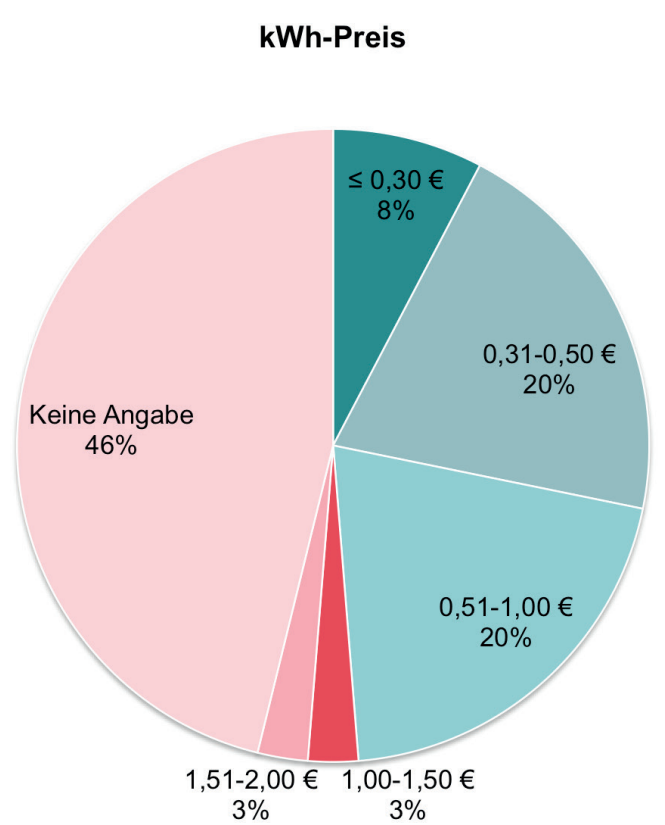

Quelle: Kopatz et al. 2017.

Prepaidzählers stärker mit ihrem Stromverbrauch zu befassen. Damit geht die für die NutzerInnen kostentransparente Wirkung des Prepaidzählers einher. Eine Kostentransparenz ist durch die Anzeige des Guthabens auf dem Display beim derzeitigen Einsatz von Prepaidzählern jedoch nur scheinbar gegeben. Die Befragung ergab, dass die NutzerInnen teilweise extrem hohe Ausgaben für Strom verzeichnen. Grund dafür ist nicht etwa ein hoher Verbrauch, sondern die Zusammenführung verschiedener Funktionen bei der Nutzung des Prepaidzählers durch die Energieversorger. So wird der Zähler meist zur Tilgung von bestehenden Altschulden von Strom und Gas durch einen erhöhten Arbeitspreis verwendet, welcher durch bestehende Altschulden zustande kommt und $\mathrm{zu}$ deutlich höheren sowie unterschiedlichen Arbeitspreisen im Vergleich zum üblichen Grundversorgungstarif führt (Abb. 3, links). Zusätzlich wird der Prepaidzähler vereinzelt für den Bezug von Gas mit dem Stromverbrauch vermischt. Diese Vermischung der Funktionen ist problematisch und führt zu einer Intransparenz hinsichtlich der Stromkosten. Zwar wissen die NutzerInnen, wie viel Guthaben sie zur Verfügung haben, jedoch bleibt unklar, wie hoch der Anteil ist, der zur Schuldentilgung oder für andere Medien verbraucht wurde. Es sind vor allem diese Aspekte, die bei den KundInnen hohe Kosten verur-

\section{Verbesserung der Beurteilung des} Verbrauchs einzelner Geräte

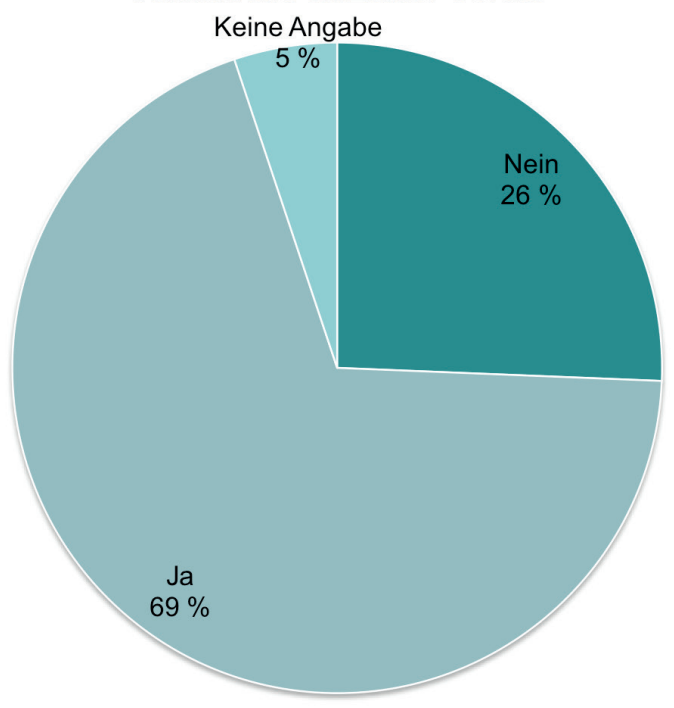

sachen und den Prepaidzähler zu einem Symbol für neoliberale Konsum- und Vertriebslogik machen, bei dem ethische Aspekte in den Hintergrund treten (Berger 2017: 421). Die Tatsachen, dass gerade einkommensarme Haushalte mehr bezahlen als reiche und dass der aktuelle Ordnungsrahmen es zudem ermöglicht, auch andere Medien sowie in unbegrenzter Höhe Altschulden über den Zähler abzurechnen, verdeutlichen, dass das Machtverhältnis zwischen VerbraucherInnen und Energieunternehmen durch diese Zähler neu konfiguriert wird (Berger 2017: 403).

Besonders bemerkenswert sind die durch die NutzerInnen erworbenen Kenntnisse bzw. Lerneffekte bezogen auf die Kosten einzelner Anwendungen. Einhergehend mit der Nutzung des Prepaidzählers ist eine Verbesserung der Beurteilung des Verbrauchs einzelner Geräte durch die NutzerInnen (Abb. 3 rechts). Kontrollieren sie beispielsweise vor und nach dem Gebrauch der Waschmaschine das Guthaben am Display, leiten sie daraus die Kosten für den Waschvorgang ab. Viele NutzerInnen erzählten von ihren Stromkostenkalkulationen aufgrund der Anzeige im Display, einzelne führen sogar ein „Stromtagebuch“. So waren einer Nutzerin beispielsweise die Stromkosten für die Zubereitung eines Weihnachtsessens bekannt, was sie bei ihrer privaten Budgetplanung entsprechend berücksichtigt. 
3.3 Strategien der befragten Haushalte zur Stromeinsparung

Aus der Kenntnis der Stromausgaben haben die Befragten eigene Strategien entwickelt, um ihre Kosten zu senken. Dabei kommen sowohl Effizienz- als auch Suffizienzstrategien zum Tragen. Die häufigste Effizienzmaßnahme der Befragten ist der Einsatz von LED-Leuchten. Des Weiteren wurden, soweit finanziell möglich, elektrische Geräte durch effizientere ersetzt. Bei den Suffizienzmaßnahmen kommen sowohl Reduktions- als auch Substitutions- und Anpassungsstrategien zum Tragen. So schalten die interviewten Haushalte teilweise ihre Geräte komplett aus oder nutzen diese weniger häufig und kürzer. Eine Befragte gab an, die Playstation-Zeiten ihrer Kinder zu reduzieren und bemerkte bereits die daraus resultierende Kostenersparnis. Das Suffizienzverhalten der NutzerInnen kann jedoch auch problematische Dimensionen annehmen. Eine Selbstbeschränkung in der Nutzung von Licht, indem elektrisch betriebene Lampen durch Kerzen ersetzt werden, ist eine solche Anpassungsstrategie, in deren Folge es schon zu Hausbränden mit Todesfolgen kam. Derartige Maßnahmen sind somit Ausdruck eines regelrechten Ausschlusses von einem menschenwürdigen Leben und keine sinnvolle Maßnahme zur Energieeinsparung. Denn hierbei handelt es sich um regelrecht erzwungene Einsparmaßnahmen, die in erster Linie der prekären Situation des Haushalts geschuldet sind. Die PrepaidnutzerInnen passen sich somit in solchen Fällen, um Kosten zu sparen, an Konditionen unterhalb eines „normalen“ Lebensstils an (vgl. Brunner et al. 2012), was auch mit erheblichen Risiken für die Gesundheit verbunden sein kann. In Neuseeland, wo Elektrizität der häufigste Energieträger für das Heizen ist, sind vor allem SeniorInnen gesundheitlich von Energiearmut geschädigt, was sich sogar in einer steigenden Mortalitätsrate in der saisonalen Heizperiode zeigt (Berger 2017: 412). Auch wir konnten im Rahmen unserer zwischen November und Januar 2016/17 durchgeführten Hausbesuche feststellen, dass (obwohl nicht mit Strom geheizt wurde) in den Wohnungen teilweise sehr geringe Temperaturen herrschten und unsere InterviewpartnerInnen warm angezogen waren (Kopatz et al. 2017: 53 f.).

Durch die Nutzung eines Prepaidzählers konnten sich bei den Befragten Stromeinspareffekte einstellen. 51 Prozent der befragten Haushalte gaben an, mit dem Prepaidzähler einen geringeren Stromverbrauch zu haben als mit einem konventionellen Stromzähler. Ein
Grund dafür ist vermutlich die verstärkte Beschäftigung mit dem Stromverbrauch im Haushalt. 79 Prozent der befragten Haushalte verzeichneten eine stärkere Beschäftigung seit dem Einsatz des Prepaidzählers. Gleichwohl zeigt die Befragung, dass noch deutlich mehr Hilfestellungen zur Erschließung von Stromspareffekten angenommen würden.

\section{Fazit und Ausblick}

Prepaidzähler haben aus Perspektive der KundInnen eine Vielzahl von Vor- und Nachteilen. $\mathrm{Zu}$ den wesentlichen Vorteilen zählt, dass Haushalte durch den Umstieg auf einen Prepaidzähler eine Stromsperre haben vermeiden können oder bei bereits bestehender Stromsperre eine Wiederaufnahme der Stromversorgung erfolgen kann. Zudem führt die subjektiv gestiegene Transparenz des Stromverbrauchs durch die engmaschige Kontrollmöglichkeit des Guthabens zur Steigerung der Energieeffizienz und -suffizienz. Ein geringerer Stromverbrauch führt, sofern keine überhöhten Gebühren anfallen, zu niedrigeren Ausgaben für Energie und stabilisiert so die soziale Lage von einkommensarmen Haushalten. Energieschulden können vermieden werden, wodurch auch die Versorger im Inkassobereich einen Vorteil haben. Die von Kritikern häufig vorgebrachten Vorbehalte gegenüber Prepaidzählern, wie die soziale Stigmatisierung in Mietshäusern und mit einer Selbstsperre einhergehende Probleme, konnten durch unsere Befragungen nicht bestätigt werden. Im Gegenteil, die Zufriedenheit der NutzerInnen ist trotz des höheren Aufwands sehr hoch. Auch eine kürzlich in Österreich durchgeführte Untersuchung zeigt, dass die Betroffenen erste Vorbehalte schnell abgebaut und auch nach der Rückzahlung von Altschulden oft der Wunsch geäußert wurde, den Prepaidzähler behalten zu wollen (Stückler 2015: 28). Jedoch macht unsere Untersuchung auch Schattenseiten deutlich: In Deutschland werden immer mehr Prepaidzähler installiert, für die Nutzung jedoch gibt es bislang keinen gesetzlichen Ordnungsrahmen. Dementsprechend schafft sich jeder Prepaid-anbietende Energieversorger seine eigenen Regularien. So belegt unsere Studie, dass Stromanbieter auch Altschulden in Form eines erhöhten Arbeitspreises abrechnen, teilweise findet sogar eine gemeinsame Schuldentilgung von Strom- und Gasschulden über den Zähler statt. Die NutzerInnen sehen zwar auf dem Display des Zählers, wie viel Geld noch für den Stromverbrauch zur Verfügung steht, die vermeintliche Transparenz ist 
durch die genannten Abrechnungsmethoden jedoch nur scheinbar vorhanden und die gekoppelte Abrechnung ist als irreführend zu bewerten. Insbesondere hinsichtlich der Tarife ist daher eine Rahmenvorgabe zu empfehlen, die den Umgang mit Altschulden und die Höhe der Kosten für VerbraucherInnen regelt. Die Bereitstellung von notwendiger Energie für ein „normales" Leben ist ein zentraler Aspekt der Daseinsvorsorge, der momentan für den Bereich Prepaid in Deutschland nicht geregelt ist, und das, obwohl die betroffenen Haushalte in einem besonderen Abhängigkeitsverhältnis zum Energieversorgungsunternehmen stehen. Insbesondere hinsichtlich des Rechts der VerbraucherInnen, durch den Einbau eines Prepaidzählers eine Stromsperre vermeiden zu können und dem gleichzeitigen Schutz vor überhöhten Tarifen könnten für Deutschland die in Österreich eingeführten gesetzlichen Regelungen Vorbild sein. Vor Inkrafttreten der österreichischen Gesetzesinitiative wurden den Betroffenen Beträge von bis zu 250 Euro für den Einbau in Rechnung gestellt, jetzt sind es nur noch 20 Euro, die für Installation und etwaige Deinstallation verlangt werden können. Erhöhte Tarife sind verboten (Berger 2017: 415f.). Das österreichische Elektrizitätswirtschafts- und -organisationsgesetz von 2010 belegt damit, dass eine ordnungsrechtliche Vorgabe in Form von Höchstpreisregelungen ein erfolgversprechender Weg zum Schutz einer besonders vulnerablen Haushaltsgruppe sein kann.

Insgesamt kann festgestellt werden, dass in Deutschland eine erste Problemsensibilisierung stattfindet. Im Landesmodellprojekt „NRW bekämpft Energiearmut" tritt beispielsweise die Verbraucherzentrale NRW gemeinsam mit örtlichen Grundversorgern und der Caritas in einem durch das Land NRW finanzierten Projekt dem wachsenden Problem rund um Energiearmut und Energiesperren entgegen (Schöllgen \& Kosbab 2017: 475 ff).

Die Ergebnisse unserer Studie lassen darauf schließen, dass die Prepaidzähler Stromspareffekte bewirken. Dieses Ergebnis basiert jedoch nicht auf gemessenen Werten, sondern auf den subjektiven Einschätzungen der interviewten Haushalte. Daher ist bislang unbekannt, wie hoch die Einsparungen tatsächlich sind und welchen Anteil Suffizienzstrategien bzw. bewusste "Einsparinvestitionen" und Effizienzmaßnahmen ausmachen. Bei den erzielten Stromeinsparungen, die auf Verhaltensänderungen zurückzuführen sind, wäre es aus sozialen Gründen wichtig zu erfahren, ob diese mit starken Komforteinbußen, Unannehmlichkeiten oder Entbehrungen einhergehen. Es besteht daher weiterer Forschungsdarf hinsichtlich Art und Umfang des Stromeinspareffekts durch Prepaidzähler.

\section{Literatur}

Berger, T. (2011): Energiearmut: Eine Studie über Situation, Ursachen, Betroffene AkteurInnen und Handlungsoptionen. Wien: Spectro gemeinnützige Gesellschaft für wissenschaftliche Forschung GmbH.

Berger, T. (2017): Energie prepaid. Sozio-technische Implikationen im Management energiearmer KonsumentInnen durch Prepayment-Meter. In: Großmann, K./Schaffrin, A./Smigiel, C. (Hg.): Energie und soziale Ungleichheit. Zur gesellschaftichen Dimension der Energiewende in Deutschland und Europa. Wiesbaden: Springer, 403-424.

Bortz, J./Döring, N. (1995): Forschungsmethoden und Evaluation für Sozialwissenschaftler. Berlin/Heidelberg: Springer.

Brischke, L.-A./Thomas, S. (2014): Energiesuffizienz im Kontext der Nachhaltigkeit. Definition und Theorie. Arbeitspapier im Rahmen des Projektes „Strategien und Instrumente für eine technische, systemische und kulturelle Transformation zur nachhaltigen Begrenzung des Energiebedarfs im Konsumfeld Bauen / Wohnen“. ifeu, Heidelberg / Berlin; Wuppertal Institut, Wuppertal.

Brunner, K.-M./Spitzer, M./Christanell, A. (2012): Experiencing fuel poverty. Coping Strategies of low-income households in Vienna/Austria. Energy Policy, 49, 53-59.

Brutscher, P.-B. (2012): Self-Disconnection Among PrePayment Customers - A Behavioural Analysis. Online: http://www.eprg.group.cam.ac.uk/wp-content/uploads/2014/o1/EPRG-Complete_1207.pdf [09.10.2017].

Bundeskartellamt/Bundesnetzagentur (2016): Monitoringbericht 2016. Bonn.

Der Paritätische Gesamtverband (2017): Menschenwürde ist Menschenrecht. Bericht zur Armutsentwicklung in Deutschland 2017. Berlin.

E-Control (2013): Energiearmut in Österreich, Definitionen und Indikatoren. Wien: Energie-Control Austria.

European Commission (2016): Consumer vulnerability across key markets in the European Union - Final report. Brüssel: EC.

Faruqui, A./Sergici, S./Sharif, A. (2010): The impact of informational feedback on energy consumption - A survey of the experimental evidence. Energy, 35 (4), 1598-1608.

Großmann, K. (2017): Energiearmut als multiple Deprivation vor dem Hintergrund diskriminierender Systeme. In: Großmann, K./Schaffrin, A./Smigiel, C. (Hg.): Energie und soziale Ungleichheit. Zur gesellschaftlichen Dimension der Energiewende in Deutschland und Europa. Wiesbaden: Springer, $55-78$.

Heindl, P. (2014): Measuring Fuel Poverty: General Considerations and Application to German Household Data. Zen- 
trum für Europäische Wirtschaftsforschung: Discussion Paper No. 13-04. Mannheim.

Heindl, P./Löschel, A. (2016): Analyse der Unterbrechungen der StromGVV. Gutachten im Auftrag des Bundesministeriums für Wirtschaft und Energie. Online: http://ftp. zew.de/pub/zew-docs/gutachten/AnalyseUnterbrechungenStromversorgung2016.pdf [09.10.2017].

Hodges, N./Roberts, S./Smith, K./Bridgeman, T./Banks, N./ Asher, M. (2016): Smart prepayment and fuel poverty. A report for eaga charitable trust. Bristol: Centre for Sustainable Energy.

Kopatz, M./Spitzer, M./Christanell, A. (2010): Energiearmut. Stand der Forschung, national Programme und regionale Modellprojekte in Deutschland, Österreich und Großbritannien. Wuppertal Papers - Wuppertal Institut für Klima, Umwelt und Energie.

Kopatz, M. (2013): Energiewende, Aber fair! Wie sich die Energiezukunft tragfähig gestalten lässt. München: Oekom Verlag.

Kopatz, M./Wagner, O./Drissen, I./Wiegand, J./Theuer, L. (2017): Guthabenzahlung für Strom: Studie über den Breiteneinsatz von Prepaidzählern. Wuppertal Report 11. Wuppertal Institut für Klima, Umwelt, Energie, Wuppertal.

ofgem (2017): Statutory consultation for a vulnerable customer safeguard tariff. Pressemitteilung vom 11. Oktober 2017. Online: https://www.ofgem.gov.uk/system/files/ docs/2017/10/statutory_consultation_for_a_vulnerable_customer_safeguard_tariff.pdf [09.10.2017].

O'Sullivan, K. C./Howden-Chapman, P. L./Fougere, G. M./ Hales, S./Stanley, J. (2013): Empowered? Examining selfdisconnection in a postal survey of electricity prepayment meter consumers in New Zealand. Energy Policy, 52, 277-287. Online: http://www.sciencedirect.com/science/article/pii/So301421512007951 [09.10.2017].

O’Sullivan, K. C./Stanley, J./Fougere, G./Howden-Chapman, P. L. (2016): Heating practices and self-disconnection among electricity prepayment meter consumers in New Zealand: A follow-up survey. Utilities Policy, 41, 139-147. Online: http://www.sciencedirect.com/science/article/ pii/So957178716301862 [09.10.2017].

PWC (2015): Bevölkerungsbefragung Stromanbieter 2015. Online: https://www.pwc.de/de/energiewirtschaft/assets/ pwc-umfrage-energie.pdf [04.12.2017].

Sachverständigenrat für Umweltfragen (2016): SRU-Umweltgutachten 2016: Impulse für eine integrative Umweltpolitik. Berlin.

Savage, M./Devine, F./Cunningham, N./Taylor, M./Li, Y./ Hjellbrekke, J./Le Roux, B./Friedman, S./Miles, A. (2014): A new model of social class? Findings from the BBC's Great British Class Survey experiment. Sociology, 47 (2), 219-25o. Online: http://eprints.lse.ac.uk/49654/1/ Savage_New_model_social_class_2014.pdf [09.10.2017].

Schaffrin, A/ Smigiel, C./ Großmann, K (2017): Energie und soziale Ungleichheit in Deutschland und Europa - eine Einführung. In: Großmann, K./Schaffrin, A./Smigiel, C. (Hg.): Energie und soziale Ungleichheit. Zur gesellschaft- lichen Dimension der Energiewende in Deutschland und Europa. Wiesbaden: Springer, 1-26.

Schnell, R./Hill, P. B./Esser E. (1989): Methoden der empirischen Sozialforschung. München/Wien: Oldenbourg Wissenschaftsverlag.

Schöllgen C./Kosbab S. (2017): Energiesperren vermeiden, Energiearmut lindern. Erfahrungen aus NordrheinWestfalen. In: Großmann, K./Schaffrin, A./Smigiel, C. (Hg.): Energie und soziale Ungleichheit. Zur gesellschaftlichen Dimension der Energiewende in Deutschland und Europa. Wiesbaden: Springer, 475-492.

Simpson, K./Smith, K./Thomas, K. (2016): Smart prepayment meters: householder experiences. Final report to Chesshire Lehmann Fund, March 2016. Bristol: Centre for Sustainable Energy.

Stadtwerke Duisburg (2017): PartnerStrom Prepaid. Online: https://www.stadtwerke-duisburg.de/privatkunden/ strom/partnerstrom-prepaid/ [09.10.2017].

Stier, W. (1999): Empirische Forschungsmethoden. Berlin/Heidelberg: Springer.

Stückler, H. (2015): Energieberatung einkommensschwacher Haushalte. Graz: Energie Agentur Steiermark.

Strünck, C. (2017): Energiearmut bekämpfen - Instrumente, Maßnahmen und Erfolge in Europa. Friedrich-EbertStiftung, Abteilung Wirtschafts- und Sozialpolitik (Hg.), Bonn.

Verbraucherzentrale NRW (2008): Vorschlag der Verbraucherzentrale NRW zur Einführung eines Strom-Spartarifes („Sozialtarif“) für private Haushalte. Düsseldorf.

Wagner, O./Richter, N./Berlo, K./Thomas, S./Irrek, K./ Seifried, D. (2008): Kurzgutachten für das Bundesministerium für Ernährung, Landwirtschaft und Verbraucherschutz (BMELV) zur Bewertung einer möglichen Veränderung der Stromtarifstruktur für Haushaltskunden („Stromspartarif“). Wuppertal Institut, Wuppertal. Online: https://epub.wupperinst.org/frontdoor/index/ index/docId/3134 [01.12.2017]. 